\title{
Intracranial Pressure versus Phase-Contrast MR Imaging for Normal Pressure Hydrocephalus
}

$\mathbf{T}$ he article by Ringstad et al, ${ }^{1}$ in this issue of the American Journal of Neuroradiology suggests that invasive intracranial pressure monitoring is a better way to select patients for shunting for normal pressure hydrocephalus (NPH) than phase-contrast MR imaging. The authors advocated placing an intracranial pressure (ICP) monitor through a burr-hole into the brain parenchyma and measuring the mean amplitude of the ICP waves (MWA) for 8 hours while the patient is sleeping. Phase-contrast MR imaging (PCMR), on the other hand, is performed noninvasively in a few minutes to determine the aqueductal CSF stroke volume (ASV). ${ }^{2}$ This technique, when properly performed, allows the determination of the ASV, which is the average of the craniocaudal and caudocranial CSF flow volumes during systole and diastole, respectively. ${ }^{3}$

The basis of the elevated MWA is decreased intracranial compliance (ie, a stiffer brain, which is 1 feature of NPH). The basis of an elevated ASV is a combination of enlarged ventricles and no atrophy. Because NPH eventually leads to atrophy, the ASV decreases and the patient is less likely to respond to ventriculoperitoneal shunting.

The authors divided 21 patients with clinical NPH into 2 groups: "shunt" $(n=17)$ and "conservative" $(n=4)$ based on MWA elevation. Sixteen of the 17 patients with shunts responded favorably during a year of follow-up, improving their NPH scores from 10 to 12 (zero being the worst and 15 being the best), while the 4 conservative patients without shunts worsened from 11 to 8 (Table 1). The authors used their finding that the ASV was higher in the unshunted conservative group (Table 2) to denigrate the value of PCMR. An alternate explanation of these data might be that MWA elevation was not useful in distinguishing the patients who should have been shunted from those who would not have benefited from a shunt (ie, their conservative group). On the basis of the elevated ASV, in fact, the patients in the conservative group with NPH symptoms might have benefited from a shunt rather than having their conditions deteriorate across time. As pointed out by the authors, with only 4 patients in the conservative group, this comparison is hardly statistically significant.

Our group has been using the finding of hyperdynamic CSF flow through the aqueduct as a predictor of shunt-responsive
NPH for $>30$ years. In the early days of MR imaging (1983-1984), we used a marked aqueductal CSF flow void on conventional proton-attenuation weighted images without flow compensation to select patients for shunting. We found the association of hyperdynamic CSF flow and shunt responsiveness significant $(P<$ $.003){ }^{4}$

With the subsequent ubiquitous use of flow compensation and fast spin-echo (with the rephasing effects of the multiple $180^{\circ}$ radiofrequency pulses), the CSF flow void sign became less sensitive (though it still remains highly specific). This result led us in the early 1990s to use PCMR to determine the volume of CSF flowing up or down over the cardiac cycle (ie, the ASV). ${ }^{2}$ In the mid-1990s, we evaluated 19 patients with symptoms of NPH who were subsequently shunted. ${ }^{3}$ All 13 patients with an ASV of $>42$ $\mathrm{uL}$ on that particular MR imaging system responded to shunting, while only half of the 6 patients with an ASV of $<42 \mathrm{uL}$ responded (ie, the ASV as we measured it had a $100 \%$ positive predictive value). While we initially thought that all the patients with ASVs of $<42 \mathrm{uL}$ had atrophy, Scollato et $\mathrm{al}^{5}$ pointed out that some of these patients may, in fact, have been very early in their disease because their ASV 6 months later was increased.

Ringstad et $\mathrm{al}^{1}$ pointed out that some investigators have been unsuccessful in using the ASV to discriminate those patients who will respond from those who will not respond to shunting for NPH. Unfortunately, not everyone uses the same technique. Our technique $^{2,3}$ uses a $512 \times 512$ matrix over a $16-\mathrm{cm}$ FOV, yielding pixels $0.312 \mathrm{~mm}$ on a side. Larger pixels (like the $0.6 \times 0.8 \mathrm{~mm}$ used in the present study) are more susceptible to partial volume averaging of stationary tissue in the surrounding midbrain. This susceptibility may explain why Ringstad et $\mathrm{al}^{1}$ used a velocityencoding factor (VENC) of only $10 \mathrm{~cm} / \mathrm{s}$, whereas we find that we need a VENC of 10,20, and $30 \mathrm{~cm} / \mathrm{s}$ to maximize accuracy (lower VENC) and avoid aliasing (higher VENC). While the authors fit the data to a sinusoidal curve to remove aliasing when present, this fitting requires manual processing, while our technique using 3 VENCs can be processed with routine flow-analysis software (eg, ReportCARD; GE Healthcare, Milwaukee, Wisconsin; or NOVA; VasSol, River Forest, Illinois).

Some investigators have been critical of the specific value of 
42- $\mu \mathrm{L}$ ASV as a discriminator; however, this measurement is unfortunately machine- and software-dependent. To diagnose hyperdynamic CSF flow, one needs to first determine the normal ASV in the elderly population. Thus, when we start using a new MR imaging system for this determination, we always perform the measurement on 10-20 elderly patients without enlarged ventricles. We then look for a volumetric flow rate of twice the normal rate to diagnose hyperdynamic CSF flow. We have found that this correlates with shunt-responsive NPH in appropriately symptomatic patients.

In regard to using patients without enlarged ventricles to determine the normal ASV, patients who eventually develop NPH often have had enlarged ventricles for many years before symptom onset. ${ }^{6}$ We call this "pre-NPH," ${ }^{\prime 6}$ while Kato et $\mathrm{al}^{7}$ labeled it "asymptomatic ventricular enlargement with features of idiopathic NPH on MR imaging."

In our institution, our neurologists and neurosurgeons use PCMR and a high-volume tap test to determine which patients should be shunted for symptomatic NPH. However, screening techniques vary around the world. Some sites go beyond the outpatient tap test and use external lumbar drainage, which is a 3-day in-patient procedure in which $10 \mathrm{~mL}$ of CSF is removed every hour via a lumbar catheter with gait reassessment at the end. ${ }^{8}$ In Sweden, the saline infusion technique is often used to determine increased resistance to inflow. ${ }^{9}$ In Japan, a midcoronal MR imaging scan is used to look for the disproportionately enlarged subarachnoid space hydrocephalus (DESH) pattern, which includes an Evans ratio of $>0.3$, enlarged Sylvian cisterns, and a tight superior convexity subarachnoid space. ${ }^{10}$ In fact, Ishikawa et $\mathrm{al}^{11}$ have reported that the tap test adds nothing to the selection of patients with $\mathrm{NPH}$ for shunting if a DESH pattern is present. Since learning of the DESH pattern 4 years ago, we have added coronal imaging to our rule out NPH protocol. Unfortunately, we have had less success with the DESH sign than the Japanese investigators, with DESH correlating with neither ASV nor shunt response.

In summary, the diagnosis of shunt-responsive NPH seems to vary by region and specialty. As demonstrated by Ringstad et al, ${ }^{1}$ neurosurgeons seem to be comfortable with placing an ICP monitor in the brain to measure compliance. If you are a radiologist, PCMR to measure the ASV may be the preferred technique. While these tests are measuring different properties of shunt-responsive $\mathrm{NPH}$, I suspect, given the choice, that most patients would prefer the less invasive evaluation.

\section{REFERENCES}

1. Ringstad G. Emblem KE, Geier O, et al. Aqueductal stroke volume: comparisons with intracranial pressure scores in idiopathic normal pressure hydrocephalus. AJNR Am J Neuroradiol 2015 May 14. [Epub ahead of print] CrossRef Medline

2. Nitz WR, Bradley WG Jr, Watanabe AS, et al. Flow dynamics of cerebrospinal fluid: assessment with phase-contrast velocity MR imaging performed with retrospective cardiac gating. Radiology 1992; 183:395-405 CrossRef Medline

3. Bradley WG Jr, Scalzo D, Queralt J, et al. Normal-pressure hydrocephalus: evaluation with cerebrospinal fluid flow measurements at MR imaging. Radiology 1996;198:523-29 CrossRef Medline

4. Bradley WG Jr, Whittemore AR, Kortman KE, et al. Marked cerebrospinal fluid void: indicator of successful shunt in patients with suspected normal-pressure hydrocephalus. Radiology 1991;178: 459-66 CrossRef Medline

5. Scollato A, Tenenbaum R, Bahl G, et al. Changes in aqueductal CSF stroke volume and progression of symptoms in patients with unshunted idiopathic normal pressure hydrocephalus. AJNR Am J Neuroradiol 2008;29:192-97 CrossRef Medline

6. Bradley WG Jr, Bahl G, Alksne JF. Idiopathic normal pressure hydrocephalus may be a "two hit" disease: benign external hydrocephalus in infancy followed by deep white matter ischemia in late adulthood. J Magn Reson Imaging 2006;24:747-55 CrossRef Medline

7. Kato T, Iseki C, Takahashi $Y$, et al. iNPH (idiopathic normal pressure hydrocephalus) and AVIM (asymptomatic ventriculomegaly with features of iNPH on MRI) [in Japanese]. Rinsho Shinkeigaku 2010;50:963-65 CrossRef Medline

8. Marmarou A, Young HF, Aygok GA, et al. Diagnosis and management of idiopathic normal-pressure hydrocephalus: a prospective study in 151 patients. J Neurosurg 2005;102:987-97 CrossRef Medline

9. Qvarlander S, Malm J, Eklund A. CSF dynamic analysis of a predictive pulsatility-based infusion test for normal pressure hydrocephalus. Med Biol Eng Comput 2014;52:75-85 CrossRef Medline

10. Hashimoto M, Ishikawa M, Mori E, et al; Study of INPH on Neurological Improvement (SINPHONI). Diagnosis of idiopathic normal pressure hydrocephalus is supported by MRI-based scheme: a prospective cohort study. Cerebrospinal Fluid Res 2010;7:18 CrossRef Medline

11. Ishikawa M, Hashimoto M, Mori E, et al. The value of the cerebrospinal fluid tap test for predicting shunt effectiveness in idiopathic normal pressure hydrocephalus. Fluids Barriers CNS 2012;9:1 CrossRef Medline

$$
\begin{array}{r}
\text { W.G. Bradley Jr } \\
\text { Department of Radiology } \\
\text { University of California San Diego Health System } \\
\text { San Diego, California }
\end{array}
$$

http://dx.doi.org/10.3174/ajnr.A4507 